\title{
DISTINGUISHING PERFECT SET PROPERTIES IN SEPARABLE METRIZABLE SPACES
}

\author{
ANDREA MEDINI
}

\begin{abstract}
All spaces are assumed to be separable and metrizable. Our main result is that the statement "For every space $X$, every closed subset of $X$ has the perfect set property if and only if every analytic subset of $X$ has the perfect set property" is equivalent to $\mathfrak{b}>\omega_{1}$ (hence, in particular, it is independent of ZFC). This, together with a theorem of Solecki and an example of Miller, will allow us to determine the status of the statement "For every space $X$, if every $\boldsymbol{\Gamma}$ subset of $X$ has the perfect set property then every $\boldsymbol{\Gamma}^{\prime}$ subset of $X$ has the perfect set property" as $\boldsymbol{\Gamma}, \boldsymbol{\Gamma}^{\prime}$ range over all pointclasses of complexity at most analytic or coanalytic.

Along the way, we define and investigate a property of independent interest. We will say that a subset $W$ of $2^{\omega}$ has the Grinzing property if it is uncountable and for every uncountable $Y \subseteq W$ there exists an uncountable collection consisting of uncountable subsets of $Y$ with pairwise disjoint closures in $2^{\omega}$. The following theorems hold.

(1) There exists a subset of $2^{\omega}$ with the Grinzing property.

(2) Assume $\mathrm{MA}+\neg \mathrm{CH}$. Then $2^{\omega}$ has the Grinzing property.

(3) Assume $\mathrm{CH}$. Then $2^{\omega}$ does not have the Grinzing property.

The first result was obtained by Miller using a theorem of Todorčević, and is needed in the proof of our main result.
\end{abstract}

All spaces are assumed to be separable and metrizable, but not necessarily Polish. See Section 1 for notation and terminology.

Definition 1. Let $X$ be a space and $\boldsymbol{\Gamma}$ a pointclass. We will say that $X$ has the perfect set property for $\boldsymbol{\Gamma}$ subsets (briefly, the $\operatorname{PSP}(\boldsymbol{\Gamma})$ ) if every $\boldsymbol{\Gamma}$ subset of $X$ is either countable or it contains a copy of $2^{\omega}$.

Notice that the $\operatorname{PSP}(\boldsymbol{\Gamma})$ gets stronger as $\boldsymbol{\Gamma}$ gets bigger.

One of the classical problems of descriptive set theory consists in determining for which pointclasses $\boldsymbol{\Gamma}$ the statement "Every Polish space has the PSP $(\boldsymbol{\Gamma})$ " holds. The following three famous theorems essentially solve this problem (see 4, Theorem 12.2(c)], 4, Theorem 13.12], and [4, Proposition 27.5] respectively).

Theorem 2 (Suslin). Every Polish space has the PSP(analytic).

Theorem 3 (Gödel). Assume $\mathrm{V}=\mathrm{L}$. Then no uncountable Polish space has the PSP(coanalytic).

Theorem 4 (Davis). Assume the axiom of Projective Determinacy. Then every Polish space has the PSP(projective) 1$]$

Date: August 9, 2014.

The author acknowledges the support of the FWF grant I 1209-N25.

${ }^{1}$ Since the axiom of Projective Determinacy has a fairly high consistency strength, it might be worth noting that an inaccessible cardinal is enough to obtain the consistency of "Every Polish space has the PSP(projective)" (this is due to Solovay, see [2, Theorem 26.14(ii)]). 
But what happens in arbitrary (that is, not necessarily Polish) spaces? By the following simple proposition, the problem described above becomes trivial.

Proposition 5. Let $X$ be a Bernstein set in some uncountable Polish space. Then $X$ does not have the $\operatorname{PSP}(\boldsymbol{\Gamma})$ for any poinclass $\boldsymbol{\Gamma}$.

Proof. Let $\boldsymbol{\Gamma}$ be a pointclass. Then $X$ itself is an uncountable $\boldsymbol{\Gamma}$ subset of $X$ that does not contain any copy of $2^{\omega}$.

Much less trivial, however, is to determine exactly for which pointclasses $\boldsymbol{\Gamma}, \boldsymbol{\Gamma}^{\prime}$ the $\operatorname{PSP}(\boldsymbol{\Gamma})$ implies the $\operatorname{PSP}\left(\boldsymbol{\Gamma}^{\prime}\right)$. More precisely, we will investigate the status of the statement "For every space $X$, if $X$ has the $\operatorname{PSP}(\boldsymbol{\Gamma})$ then $X$ has the $\operatorname{PSP}\left(\boldsymbol{\Gamma}^{\prime}\right)$ " as $\boldsymbol{\Gamma}, \boldsymbol{\Gamma}^{\prime}$ range over all pointclasses of complexity at most analytic or coanalytic. The reason for this limitation on the complexity lies in Theorem 2 and Theorem 3 , but the investigation could be carried further (see Section 6).

While Theorem 27 gives a complete solution to this problem, we will focus first on a special case, which turned out to be the most interesting one. More specifically, we will consider the statement "For every space $X$, if $X$ has the PSP(closed) then $X$ has the PSP(analytic)". As we will show in Section 3, this statement is in fact equivalent to $\mathfrak{b}>\omega_{1}$ (see Theorem[14). We will need an auxiliary property, which is defined and investigated in Section 2. In Section 4, we will complement Theorem 14 by establishing all results relevant to our problem that can be proved in ZFC alone. Finally, in Section 5 and Section 6, we will investigate natural generalizations of the properties that we considered and state several open questions.

To the reader interested in more instances of "dropping Polishness", we recommend the articles [9] and [11] of Miller, which partly inspired our work.

\section{TeRminology AND NOtATION}

We will generally follow [5]. However, since the spaces that we will deal with are not necessarily Polish, we prefer to recall the relevant notions and a few basic facts about them. Throughout this article, $\boldsymbol{\Gamma}$ will always denote one of the following (boldface) pointclasses.

- $\boldsymbol{\Sigma}_{\xi}^{0}$ or $\boldsymbol{\Pi}_{\xi}^{0}$, where $\xi$ is an ordinal such that $1 \leq \xi<\omega_{1}$ (these are the Borel pointclasses).

- $\boldsymbol{\Sigma}_{n}^{1}$ or $\boldsymbol{\Pi}_{n}^{1}$, where $n$ is an ordinal such that $1 \leq n<\omega$ (these are the projective pointclasses).

We will assume that the definition of a $\boldsymbol{\Gamma}$ subset of a Polish space is well-known, and recall that it can be generalized to arbitrary spaces as follows. In the case of the Borel pointclasses, this is not really necessary, because the usual definition works in arbitrary spaces (see for example [5, Section 11.B]). However, we prefer to give a unified treatment.

Definition 6. Fix a pointclass $\Gamma$. Let $X$ be a space. We will say that $A \subseteq X$ is a $\boldsymbol{\Gamma}$ subset of $X$ if there exists a Polish space $T$ containing $X$ as a subspace such that $A=B \cap X$ for some $\boldsymbol{\Gamma}$ subset $B$ of $T$.

It is easy to check that, when $\boldsymbol{\Gamma}$ is a Borel pointclass, the above definition coincides with the usual one. We will denote $\boldsymbol{\Sigma}_{1}^{0}$ by open, $\boldsymbol{\Pi}_{1}^{0}$ by closed, $\boldsymbol{\Sigma}_{2}^{0}$ by $\boldsymbol{F}_{\sigma}, \boldsymbol{\Pi}_{2}^{0}$ by $\mathrm{G}_{\delta}, \boldsymbol{\Sigma}_{1}^{1}$ by analytic, $\boldsymbol{\Pi}_{1}^{1}$ by coanalytic. We will also denote $\bigcup_{1 \leq \xi<\omega_{1}} \boldsymbol{\Sigma}_{\xi}^{0}$ by Borel and $\bigcup_{1 \leq n<\omega} \Sigma_{n}^{1}$ by projective. 
The following "reassuring" proposition can be proved by induction on $\boldsymbol{\Gamma}$. Since every space is homeomorphic to a subspace of $[0,1]^{\omega}$, one can assume without loss of generality that $T$ is Polish. This makes it possible to use Lavrentiev's Theorem (see [5, Theorem 3.9]) in the inductive step from $\boldsymbol{\Pi}_{n}^{1}$ to $\boldsymbol{\Sigma}_{n+1}^{1}$, which is the only non-trivial part of the proof.

Proposition 7. Fix a pointclass $\boldsymbol{\Gamma}$. Let $X$ be a space and $A$ a subset of $X$. The following conditions are equivalent.

- $A$ is a $\boldsymbol{\Gamma}$ subset of $X$.

- For every space $T$ containing $X$ as a subspace there exists a $\boldsymbol{\Gamma}$ subset $B$ of $T$ such that $A=B \cap X$.

One could also define the so-called ambiguous pointclasses as follows.

- Let $\boldsymbol{\Delta}_{\xi}^{0}=\boldsymbol{\Sigma}_{\xi}^{0} \cap \boldsymbol{\Pi}_{\xi}^{0}$ for every ordinal $\xi$ such that $1 \leq \xi<\omega_{1}$.

- Let $\boldsymbol{\Delta}_{n}^{1}=\boldsymbol{\Sigma}_{n}^{1} \cap \boldsymbol{\Pi}_{n}^{1}$ for every ordinal $n$ such that $1 \leq n<\omega$.

However, it is not hard to realize that they would not add any interesting content to our results, while making the exposition more cumbersome. Therefore, we will exclude them from our discussion. The only exception is $\boldsymbol{\Delta}_{1}^{0}$, which we will denote by clopen (see Proposition 26] and Theorem 27).

Our reference for topology will be [8]. By $X \approx Y$ we will mean that the spaces $X$ and $Y$ are homeomorphic. In this case, we will say that $Y$ is a copy of $X$. Given an infinite cardinal $\kappa$, we will say that a space $X$ is $\kappa$-crowded if it is non-empty and every non-empty open subset of $X$ has size at least $\kappa$. Recall that a sequence $\left\langle A_{n}: n \in \omega\right\rangle$ of subsets of a space $X$ converges to a point $x \in X$ if for every neighborhood $U$ of $x$ there exists $m \in \omega$ such that $A_{n} \subseteq U$ whenever $n \geq m$.

Recall that a subset $B$ of an uncountable Polish space $T$ is a Bernstein set if $B \cap K \neq \varnothing$ and $(T \backslash B) \cap K \neq \varnothing$ for every copy $K$ of $2^{\omega}$ in $T$. It is easy to see that Bernstein sets exist in ZFC. Since $2^{\omega} \approx 2^{\omega} \times 2^{\omega}$, every Bernstein set has size $\mathfrak{c}$.

Recall that a subset $L$ of a Polish space $T$ is a Luzin set if it is uncountable and every uncountable subset of $L$ is non-meager in $T$. It is easy to see that Luzin sets exist under the assumption $\operatorname{cof}(\mathrm{BP})=\omega_{1}$.

Our reference for pure set theory will be 2 . Given $f, g \in \omega^{\omega}$, we will write $f<^{*} g$ if there exists $m \in \omega$ such that $f(n)<g(n)$ for every $n \geq m$. Recall that a family $B \subseteq \omega^{\omega}$ is unbounded if there exists no $g \in \omega^{\omega}$ such that $f<^{*} g$ for every $f \in B$. We will denote by $\mathfrak{b}$ the least size of an unbounded family in $\omega^{\omega}$.

\section{The Grinzing PRoperty}

In this section we investigate the property defined as follows. The only result from this section that will be used in the remainder of the article (namely, in the proof of Proposition [19) is Corollary 11. However, we believe that Proposition 12 and Theorem 13 are of independent interest. See also Section 5 .

Definition 8. We will say that a subset $W$ of $2^{\omega}$ has the Grinzing property 2 (briefly, the GP) if it is uncountable and for every uncountable $Y \subseteq W$ there exist uncountable subsets $Y_{\alpha}$ of $Y$ for $\alpha \in \omega_{1}$ such that $\operatorname{cl}\left(Y_{\alpha}\right) \cap \mathrm{cl}\left(Y_{\beta}\right)=\varnothing$ whenever $\alpha \neq \beta$, where the closure is taken in $2^{\omega}$.

\footnotetext{
${ }^{2}$ This property is named after a lovely area of Vienna, in which the author lived while this article was being written.
} 
Notice that an uncountable subset $W$ of $2^{\omega}$ has the GP if and only if every subset of $W$ of size $\omega_{1}$ has the GP.

An early version of this article contained an erroneous proof of Corollary 11 Miller spotted the mistake and supplied a new proof, based on Theorem 10. We will need the following definition. Given a subtree $\mathcal{T}$ of $\omega^{<\omega_{1}}$ and $\alpha<\omega_{1}$, let $\mathcal{T}_{\alpha}=\mathcal{T} \cap \omega^{\alpha}$ denote the $\alpha$-th level of $\mathcal{T}$. Also let $\mathcal{T}_{<\gamma}=\bigcup_{\alpha<\gamma} \mathcal{T}_{\alpha}$ for every limit ordinal $\gamma<\omega_{1}$.

Definition 9 (Todorčević). We will say that $\left\langle K_{s}: s \in \mathcal{T}\right\rangle$ is an Aronszajn tree of perfect sets if the following conditions are satisfied.

(1) $\mathcal{T}$ is a subtree of $\omega^{<\omega_{1}}$.

(2) $\mathcal{T}_{\alpha}$ is non-empty and countable for every $\alpha \in \omega_{1}$.

(3) $K_{s}$ is a perfect subset of $2^{\omega}$ for every $s \in \mathcal{T}$.

(4) $K_{t} \subsetneq K_{s}$ whenever $t \supsetneq s$.

(5) $K_{s} \cap K_{t}=\varnothing$ whenever $t \perp s$.

Using the fact that there are no strictly decreasing $\omega_{1}$-sequences of closed subsets of $2^{\omega}$, it is easy to check that if $\left\langle K_{s}: s \in \mathcal{T}\right\rangle$ is an Aronszajn tree of perfect sets then $\mathcal{T}$ is in fact an Aronszajn tree in the usual sense.

The proof of following result is taken from the first part of the proof of 1 , Theorem 4], and it is included for the sake of completeness. We will employ the well-known technique of fusion, for which we refer to [2, pages 244-245]. When using the ordering $\leq_{n}$, we will identify a perfect subset $K$ of $2^{\omega}$ with the corresponding subtree $p=\left\{x\lceil n: x \in K, n \in \omega\}\right.$ of $2^{<\omega}$.

Theorem 10 (Todorčević). There exists an Aronszajn tree of perfect sets.

Proof. We will construct $\mathcal{T}_{\alpha}$ and $K_{s}$ for $s \in \mathcal{T}_{\alpha}$ simultaneously by transfinite recursion, making sure that conditions (1)-(5) in Definition 9 are satisfied. Furthermore, we will make sure that the following additional condition is satisfied.

(6) Whenever $\beta<\alpha$ and $n \in \omega$, given any $s \in \mathcal{T}_{\beta}$, there exists $t \in \mathcal{T}_{\alpha}$ such that $t \supseteq s$ and $K_{t} \leq_{n} K_{s}$.

Start by setting $\mathcal{T}_{0}=\{\varnothing\}$ and $K_{\varnothing}=2^{\omega}$. The construction at a successor stage is straightforward, so we leave it to the reader. Now assume that $\gamma<\omega_{1}$ is a limit ordinal, and that $\mathcal{T}_{<\gamma}$ and $K_{s}$ for $s \in \mathcal{T}_{<\gamma}$ have already been constructed.

Let $\left\langle\alpha_{\ell}: \ell \in \omega\right\rangle$ be a strictly increasing sequence consisting of elements of $\gamma$ such that $\alpha_{\ell} \rightarrow \gamma$. Fix $s \in \mathcal{T}_{\alpha_{n}}$. We will inductively define a chain $\left\langle t_{\ell}(s): \ell \geq n\right\rangle$ such that $t_{\ell}(s) \in \mathcal{T}_{\alpha_{\ell}}$ for every $\ell \geq n$, using the inductive hypothesis (6). Start by letting $t_{n}(s)=s$. Given $t_{\ell}(s)$ for some $\ell \geq n$, choose $t_{\ell+1}(s) \in \mathcal{T}_{\alpha_{\ell+1}}$ such that $t_{\ell+1}(s) \supseteq t_{\ell}(s)$ and $K_{t_{\ell+1}(s)} \leq_{\ell+1} K_{t_{\ell}(s)}$. Now let $t(s)=\bigcup_{\ell \geq n} t_{\ell}(s)$.

Define $\mathcal{T}_{\gamma}=\left\{t(s): s \in \bigcup_{\ell \in \omega} \mathcal{T}_{\alpha_{\ell}}\right\}$. For each $t \in \mathcal{T}_{\gamma}$, fix $s \in \mathcal{T}_{\alpha_{n}}$ such that $t=t(s)$, then define $K_{t}=\bigcap_{\ell \geq n} K_{t_{\ell}(s)}$. Notice that $K_{t}$ is a perfect subset of $2^{\omega}$ by fusion. Furthermore, it is clear that this definition does not depend on the choice of $s$. Finally, it is easy to check that conditions (1)-(6) will be mantained.

Corollary 11 (Miller). There exists a subset of $2^{\omega}$ with the GP.

Proof. Let $\left\langle K_{s}: s \in \mathcal{T}\right\rangle$ be an Aronszajn tree of perfect sets. Pick $w_{s} \in K_{s}$ for every $s \in \mathcal{T}$ so that $w_{s} \neq w_{t}$ whenever $s \neq t$. Let $W=\left\{w_{s}: s \in \mathcal{T}\right\}$. We will show that $W$ has the GP. Fix an uncountable $Y \subseteq W$ and let $\mathcal{S}$ be the subtree 
of $\mathcal{T}$ generated by $\left\{s \in \mathcal{T}: w_{s} \in Y\right\}$. Assume without loss of generality that $\mathcal{S}$ is normal (that is, $\{t \in \mathcal{S}: t \supseteq s\}$ is uncountable for every $s \in \mathcal{S}$ ).

Notice that $\left\langle K_{s}: s \in \mathcal{S}\right\rangle$ is still an Aronszajn tree of perfect sets. It follows that $\mathcal{S}$ is not ccc, otherwise forcing with $\mathcal{S}$ would yield a strictly descending $\omega_{1}$-sequence of closed subsets of $2^{\omega}$, contradicting the fact that $\omega_{1}$ is preserved. So we can fix an uncountable antichain $\left\langle s_{\alpha}: \alpha \in \omega_{1}\right\rangle$ in $\mathcal{S}$. Set $Y_{\alpha}=Y \cap K_{s_{\alpha}}$ for $\alpha \in \omega_{1}$. Obviously $\operatorname{cl}\left(Y_{\alpha}\right) \cap \mathrm{cl}\left(Y_{\beta}\right)=\varnothing$ whenever $\alpha \neq \beta$, where the closure is taken in $2^{\omega}$. Furthermore, the fact that $\mathcal{S}$ is normal implies that each $Y_{\alpha}$ is uncountable.

The following two results show that the statement " $2^{\omega}$ has the GP" is independent of ZFC.

Proposition 12. Assume $\mathrm{CH}$. Then $2^{\omega}$ does not have the GP.

Proof. We will give two proofs. The first proof uses the fact that $\mathrm{CH}$ implies $\mathfrak{b}=\omega_{1}$. In particular, we can fix an unbounded family $B=\left\{f_{\alpha}: \alpha \in \omega_{1}\right\} \subseteq \omega^{\omega}$ consisting of increasing functions such that $f_{\alpha}<^{*} f_{\beta}$ whenever $\alpha<\beta$. Let $T=(\omega+1)^{\omega}$. Notice that $B \subseteq \omega^{\omega} \subseteq T \approx 2^{\omega}$. The uncountable set $B$ will witness that $T$ does not have the GP. Let

$$
T_{\infty}=\left\{s \frown\langle\omega, \omega, \ldots\rangle: s \in \omega^{<\omega}\right\} \subseteq T .
$$

Since $T_{\infty}$ is countable, it will be enough to show that $\operatorname{cl}(C) \cap T_{\infty} \neq \varnothing$ whenever $C \subseteq B$ is uncountable, where the closure is taken in $T$. Since every uncountable subset of $B$ is unbounded, this follows from Lemma 17 and Lemma 18 .

The second proof uses the fact that $\mathrm{CH}$ implies $\operatorname{cof}(\mathrm{BP})=\omega_{1}$. In particular, we can fix a Luzin set $L$ in $2^{\omega}$. The uncountable set $L$ will witness that $2^{\omega}$ does not have the GP. Assume that $L_{\alpha}$ is an uncountable subset of $L$ for every $\alpha \in \omega_{1}$. By the definition of Luzin set, no $L_{\alpha}$ can be nowhere dense. Therefore, there exist non-empty open subsets $U_{\alpha}$ of $2^{\omega}$ for $\alpha \in \omega_{1}$ such that $U_{\alpha} \subseteq \operatorname{cl}\left(L_{\alpha}\right)$ for each $\alpha$. Let $\alpha \neq \beta$ be such that $U_{\alpha} \cap U_{\beta} \neq \varnothing$. It is clear that $\operatorname{cl}\left(L_{\alpha}\right) \cap \mathrm{cl}\left(L_{\beta}\right) \neq \varnothing$.

Theorem 13. Assume $\mathrm{MA}+\neg \mathrm{CH}$. Then $2^{\omega}$ has the GP.

Proof. Fix $Y \subseteq 2^{\omega}$ of size $\omega_{1}$. Let $Y=\left\{y_{\xi}: \xi \in \omega_{1}\right\}$ be an injective enumeration. Fix uncountable subsets $Z_{\alpha}$ of $Y$ for $\alpha \in \omega_{1}$ such that $Z_{\alpha} \cap Z_{\beta}=\varnothing$ whenever $\alpha \neq \beta$. By Lemma 30, we can also assume that each $Z_{\alpha}$ is $\omega_{1}$-crowded.

Let $\mathbb{P}$ be the set of all pairs of the form $p=\langle n, F\rangle=\left\langle n^{p}, F^{p}\right\rangle$, where $n \in \omega$ and $F=\left\langle F_{\alpha}: \alpha \in \omega_{1}\right\rangle=\left\langle F_{\alpha}^{p}: \alpha \in \omega_{1}\right\rangle$ satisfy the following conditions.

(1) Each $F_{\alpha}$ is a finite subset of $Z_{\alpha}$

(2) $\operatorname{supp}(p)=\left\{\alpha \in \omega_{1}: F_{\alpha} \neq \varnothing\right\}$ is finite.

(3) $\left\{x\left\lceil n: x \in F_{\alpha}\right\} \cap\left\{x\left\lceil n: x \in F_{\beta}\right\}=\varnothing\right.\right.$ whenever $\alpha \neq \beta$.

Given $p, q \in \mathbb{P}$, define $q \leq p$ if the following conditions are satisfied.

(4) $n^{q} \geq n^{p}$.

(5) $F_{\alpha}^{q} \supseteq F_{\alpha}^{p}$ for each $\alpha$.

(6) $\left\{x\left\lceil n^{p}: x \in F_{\alpha}^{q}\right\}=\left\{x\left\lceil n^{p}: x \in F_{\alpha}^{p}\right\}\right.\right.$ whenever $\alpha \in \operatorname{supp}(p)$.

For any given $\delta, \eta \in \omega_{1}$, define

$$
D_{\delta, \eta}=\left\{p \in \mathbb{P}: y_{\xi} \in F_{\delta}^{p} \text { for some } \xi \geq \eta\right\} .
$$

In order to show that each $D_{\delta, \eta}$ is dense, fix $p \in \mathbb{P}$ and $\delta, \eta \in \omega_{1}$. Since the case $\delta \notin \operatorname{supp}(p)$ is trivial, we will assume that $\delta \in \operatorname{supp}(p)$. Fix $x \in F_{\delta}^{p}$. Notice that $Z_{\delta} \cap\left[x\left\lceil n^{p}\right]\right.$ is uncountable because $Z_{\delta}$ is $\omega_{1}$-crowded and $x \in Z_{\delta}$. In particular, 
there exists $\xi \geq \eta$ such that $y_{\xi} \in Z_{\delta}$ and $y_{\xi}\left\lceil n^{p}=x\left\lceil n^{p}\right.\right.$. Let $F^{q}=\left\langle F_{\alpha}^{q}: \alpha \in \omega_{1}\right\rangle$, where $F_{\alpha}^{q}=F_{\alpha}^{p}$ for every $\alpha \neq \delta$ and $F_{\delta}^{q}=F_{\delta}^{p} \cup\left\{y_{\xi}\right\}$. Define $q=\left\langle n^{q}, F^{q}\right\rangle$, where $n^{q} \geq n^{p}$ is such that condition (3) is satisfied. It is clear that $q \in D_{\delta, \eta}$ and $q \leq p$.

Next, we will show that $\mathbb{P}$ is ccc. Fix $\mathcal{A} \subseteq \mathbb{P}$ such that $|\mathcal{A}|=\omega_{1}$. By passing to an uncountable subset of $\mathcal{A}$, we can assume that there exists $n \in \omega$ such that $n^{p}=n$ for all $p \in \mathcal{A}$. By the Delta System Lemma, we can assume that there exists a finite $R \subseteq \omega_{1}$ such that $\operatorname{supp}(p) \cap \operatorname{supp}(q)=R$ whenever $p, q \in \mathcal{A}$ are distinct. By passing to an uncountable subset of $\mathcal{A}$ once more, we can assume that $\left\{x\left\lceil n: x \in F_{\alpha}^{p}\right\}=\left\{x\left\lceil n: x \in F_{\alpha}^{q}\right\}\right.\right.$ for every $\alpha \in R$ whenever $p, q \in \mathcal{A}$. Now fix distinct $p, q \in \mathcal{A}$ and let $F^{r}=\left\langle F_{\alpha}^{p} \cup F_{\alpha}^{q}: \alpha \in \omega_{1}\right\rangle$. Define $r=\left\langle n^{r}, F^{r}\right\rangle$, where $n^{r} \geq n$ is such that condition (3) is satisfied. It is clear that $r \in \mathbb{P}$ and $r \leq p, q$. In particular, $\mathcal{A}$ is not an antichain.

Since we are assuming $\mathrm{MA}+\neg \mathrm{CH}$ and the collection of dense sets

$$
\mathcal{D}=\left\{D_{\delta, \eta}: \delta, \eta \in \omega_{1}\right\}
$$

has size $\omega_{1}$, there exists a $\mathcal{D}$-generic filter $G \subseteq \mathbb{P}$. Define $Y_{\alpha}=\bigcup\left\{F_{\alpha}^{p}: p \in G\right\}$ for every $\alpha \in \omega_{1}$. The dense sets $D_{\delta, \eta}$ ensure that each $Y_{\alpha}$ is uncountable.

Finally, in order to get a contradiction, assume that $w \in \mathrm{cl}\left(Y_{\alpha}\right) \cap \mathrm{cl}\left(Y_{\beta}\right)$, where $\alpha \neq \beta$. Fix $p \in G$ such that $\{\alpha, \beta\} \subseteq \operatorname{supp}(p)$ and let $n=n^{p}$. Let $w_{\alpha} \in Y_{\alpha} \cap[w\lceil n]$ and $w_{\beta} \in Y_{\beta} \cap\left[w\lceil n]\right.$. There exist $q_{\alpha}, q_{\beta} \in G$ such that $w_{\alpha} \in F_{\alpha}^{q_{\alpha}}$ and $w_{\beta} \in F_{\beta}^{q_{\beta}}$. Without loss of generality, assume that $q_{\alpha}, q_{\beta} \leq p$. In particular, by condition (6), there exist $z_{\alpha} \in F_{\alpha}^{p}$ and $z_{\beta} \in F_{\beta}^{p}$ such that $z_{\alpha}\left\lceil n=w_{\alpha}\left\lceil n\right.\right.$ and $z_{\beta}\left\lceil n=w_{\beta}\lceil n\right.$. But this implies

$$
z_{\alpha}\left\lceil n=w_{\alpha}\left\lceil n=w\left\lceil n=w_{\beta}\left\lceil n=z_{\beta}\lceil n,\right.\right.\right.\right.
$$

which contradicts condition (3).

\section{THE MAIN RESUlT}

The aim of this section is to prove the following result, which is an immediate consequence of Proposition 15] and Proposition 19.

Theorem 14. The following are equivalent.

- $\mathfrak{b}>\omega_{1}$.

- For every space $X$, if $X$ has the PSP(closed) then $X$ has the PSP(analytic).

Proposition 15. Assume $\mathfrak{b}>\omega_{1}$. Let $X$ be a space with the PSP(closed). Then $X$ has the PSP(analytic).

Proof. Assume without loss of generality that $X$ is a subspace of $T=[0,1]^{\omega}$. Fix an analytic subset $A$ of $T$ such that $A \cap X$ is uncountable. We will show that $A \cap X$ contains a copy of $2^{\omega}$. Since $A$ is analytic, there exists a closed subset $C$ of $T \times \omega^{\omega}$ such that $\pi[C]=A$, where $\pi: T \times \omega^{\omega} \longrightarrow T$ is the projection on the first coordinate. Since $A \cap X$ is uncountable, it is possible to fix $Z \subseteq \omega^{\omega}$ such that $|Z| \leq \omega_{1}$ and $\pi[(T \times Z) \cap C] \cap X$ is uncountable.

By the assumption $\mathfrak{b}>\omega_{1}$, there exist compact subsets $K_{n}$ of $\omega^{\omega}$ for $n \in \omega$ such that $Z \subseteq \bigcup_{n \in \omega} K_{n}$. Observe that each $\pi\left[\left(T \times K_{n}\right) \cap C\right] \cap X$ is a closed subset of $X$ contained in $A \cap X$. Furthermore, since $\omega_{1}$ has uncountable cofinality, at least one of them must be uncountable. The fact that $X$ has the PSP(closed) concludes the proof. 
In the remainder of this section we will employ the following three lemmas, which can be safely considered folklore.

Lemma 16. Assume that $B=\left\{f_{\alpha}: \alpha \in \omega_{1}\right\} \subseteq \omega^{\omega}$ is an unbounded family such that $f_{\alpha}<^{*} f_{\beta}$ whenever $\alpha<\beta$. Then $\mathrm{cl}(C)$ is not compact whenever $C \subseteq B$ is uncountable, where the closure is taken in $\omega^{\omega}$.

Proof. Notice that every uncountable subset of $B$ is unbounded. Therefore, we can assume $C=B$ without loss of generality. Assume, in order to get a contradiction, that $\mathrm{cl}(B)$ is compact. Let $\pi_{n}: \omega^{\omega} \longrightarrow \omega$ be the projection on the $n$-th coordinate for every $n \in \omega$. Then $\pi_{n}[\mathrm{cl}(B)]$ is a compact subset of $\omega$ for each $n$. It follows that $B \subseteq \mathrm{cl}(B) \subseteq \prod_{n \in \omega} g(n)$ for some $g \in \omega^{\omega}$, which contradicts the fact that $B$ is unbounded.

Lemma 17. Assume that $B \subseteq \omega^{\omega}$ is an unbounded family. Then $\mathrm{cl}(B) \nsubseteq \omega^{\omega}$, where the closure is taken in $(\omega+1)^{\omega}$.

Proof. Observe that $\mathrm{cl}(B)$ is compact because it is a closed subset of the compact space $(\omega+1)^{\omega}$. So $\mathrm{cl}(B) \subseteq \omega^{\omega}$ is impossible by Lemma 16 .

Lemma 18. Assume that $B \subseteq \omega^{\omega}$ consists of increasing functions. Then for every $f \in \operatorname{cl}(B) \backslash \omega^{\omega}$, where the closure is taken in $(\omega+1)^{\omega}$, there exists $s \in \omega^{<\omega}$ such that $f=s \frown\langle\omega, \omega, \ldots\rangle$.

Proof. Assume, in order to get a contradiction, that $f \in \operatorname{cl}(B)$ is such that $f(m)=\omega$ and $f(n)<\omega$, where $m<n$. Notice that

$$
U=\left\{x \in(\omega+1)^{\omega}: x(m)>f(n) \text { and } x(n)=f(n)\right\}
$$

is a neighborhood of $f$ in $(\omega+1)^{\omega}$. However, the fact that $B$ consists of increasing functions implies that $U \cap B=\varnothing$, which is a contradiction.

Proposition 19. Assume $\mathfrak{b}=\omega_{1}$. Then there exists a subspace $X$ of $2^{\omega}$ that has the PSP(closed) but not the PSP(analytic).

Proof. Since $\mathfrak{b}=\omega_{1}$, we can fix an unbounded family $B=\left\{f_{\alpha}: \alpha \in \omega_{1}\right\} \subseteq \omega^{\omega}$ such that $f_{\alpha}<^{*} f_{\beta}$ whenever $\alpha<\beta$. By Corollary 11] it is possible to fix $W \subseteq 2^{\omega}$ such that $|W|=\omega_{1}$ and $W$ has the GP. Let $W=\left\{g_{\alpha}: \alpha \in \omega_{1}\right\}$ be an injective enumeration. Define $Z=\left\{\left\langle f_{\alpha}, g_{\alpha}\right\rangle: \alpha \in \omega_{1}\right\}$.

Let $T=(\omega+1)^{\omega} \times 2^{\omega}$ and notice that $T \approx 2^{\omega}$. For every $n \in \omega$, define

$$
T_{n}=\left\{x \in(\omega+1)^{\omega}: x(n)=\omega\right\} \times 2^{\omega} \subseteq T
$$

and observe that each $T_{n} \approx 2^{\omega}$. Let

$$
X=Z \cup \bigcup_{n \in \omega} T_{n} \subseteq T .
$$

Notice that $Z$ is a $\mathrm{G}_{\delta}$ subset of $X$ because each $T_{n}$ is compact. Assume, in order to get a contradiction, that $Z$ contains a copy $K$ of $2^{\omega}$. Let $\pi: \omega^{\omega} \times 2^{\omega} \longrightarrow \omega^{\omega}$ be the projection on the first coordinate. Then $\pi[K]$ is an uncountable compact subset of $B$, because $\pi \uparrow Z$ is injective and continuous. Since this contradicts Lemma 16 . it follows that the space $X$ does not have the $\operatorname{PSP}\left(\mathrm{G}_{\delta}\right)$. In particular, $X$ does not have the PSP(analytic).

Observe that each $T_{n}$ has the PSP(closed) by Theorem 2 , Therefore, in order to show that $X$ has the PSP(closed), it will be enough to prove that $\operatorname{cl}(Y) \cap \bigcup_{n \in \omega} T_{n}$ is uncountable for every uncountable $Y \subseteq Z$, where the closure is taken in $T$. Since $W$ 
has the GP, it will be enough to show that $\mathrm{cl}(Y) \cap \bigcup_{n \in \omega} T_{n}$ is non-empty for every uncountable $Y \subseteq Z$. Notice that every uncountable subset of $B$ is unbounded. Therefore, we can assume that $Y=Z$ without loss of generality. By Lemma 17 there exists $f \in(\omega+1)^{\omega} \backslash \omega^{\omega}$ and a sequence $\left\langle\alpha_{n}: n \in \omega\right\rangle$ of elements of $\omega_{1}$ such that $\left\langle f_{\alpha_{n}}: n \in \omega\right\rangle$ converges to $f$ in $(\omega+1)^{\omega}$. On the other hand, since $2^{\omega}$ is compact, there exists $g \in 2^{\omega}$ and a subsequence of $\left\langle g_{\alpha_{n}}: n \in \omega\right\rangle$ that converges to $g$ in $2^{\omega}$. It follows that the corresponding subsequence of $\left\langle\left\langle f_{\alpha_{n}}, g_{\alpha_{n}}\right\rangle: n \in \omega\right\rangle$ converges to $\langle f, g\rangle$, which is clearly an element of $\bigcup_{n \in \omega} T_{n}$.

One might wonder whether the factor $2^{\omega}$ in the definitions of $T$ and $T_{n}$ is really needed. In other words, if one defines $T=(\omega+1)^{\omega}, T_{n}=\{x \in T: x(n)=\omega\}$ for $n \in \omega$, and $Z=B$, does it follow that $\operatorname{cl}(Y) \cap \bigcup_{n \in \omega} T_{n}$ is uncountable for every uncountable $Y \subseteq Z$ ? This first attempt was originally suggested by Kunen to the author, and it obviously shaped the above proof. However, the answer is "no" in general. For example, if $B$ consists of increasing functions then $\mathrm{cl}(Y) \cap \bigcup_{n \in \omega} T_{n}$ will always be contained in $T_{\infty}=\left\{s \frown\langle\omega, \omega, \ldots\rangle: s \in \omega^{<\omega}\right\}$ by Lemma 18 In fact, this is the very issue that led to the introduction of the Grinzing property.

We conclude this section by noting that the independence of the statement "For every space $X$, if $X$ has the PSP(closed) then $X$ has the PSP(analytic)" is much easier to prove than the sharper Theorem 14, In fact, it is enough to combine Proposition 15] with the following result. We remark that, by a result of Judah and Shelah (see [3]), the assumption $\mathfrak{b}=\omega_{1}$ (or even unif $(\mathrm{BP})=\omega_{1}$ ) is not sufficient to guarantee the existence of a Luzin set.

Proposition 20 (Zdomskyy). Assume that there exists a Luzin set in $\omega^{\omega}$. Then there exists a subspace $X$ of $2^{\omega}$ that has the PSP(closed) but not the PSP(analytic).

Proof. This proof is very similar to that of Proposition 19, so we will be brief. Define $T=(\omega+1)^{\omega}$ and $T_{n}=\{x \in T: x(n)=\omega\}$ for $n \in \omega$. Let $Z=L$ be a Luzin set in $\omega^{\omega}$. Define $X=Z \cup \bigcup_{n \in \omega} T_{n} \subseteq T$. Notice that $Z$ witnesses that $X$ does not have the $\operatorname{PSP}\left(\mathrm{G}_{\delta}\right)$, because a Luzin set cannot contain any copy of $2^{\omega}$. In particular, $X$ does not have the PSP(analytic).

In order to show that $X$ has the PSP(closed), it will be enough to prove that $\operatorname{cl}(Y) \cap \bigcup_{n \in \omega} T_{n}$ is uncountable for every uncountable $Y \subseteq Z$, where the closure is taken in $T$. So fix such a $Y$. Notice that $Y$ is not nowhere dense in $\omega^{\omega}$ because $Z$ is a Luzin set. Hence $U \subseteq \mathrm{cl}(Y)$, where $U=\left\{x \in \omega^{\omega}: s \subseteq x\right\}$ for some $s \in \omega^{<\omega}$. It is easy to check that $\mathrm{cl}(U)=\{x \in T: s \subseteq x\}$, which clearly has uncountable intersection with $\bigcup_{n \in \omega} T_{n}$.

\section{The COMPlete PiCTURe}

At this point, it seems natural to ask whether finer distinctions are possible. For example, is it consistent that there exists a space with the PSP(Borel) but not the $\operatorname{PSP}$ (analytic)? Is it consistent that there exists a space with the $\operatorname{PSP}\left(\mathrm{G}_{\delta}\right)$ but not the $\operatorname{PSP}\left(\boldsymbol{\Pi}_{3}^{0}\right)$ ? We only ask for consistency results because Proposition 15 shows that there are no such examples in ZFC.

The following result of Solecki (see [12, Theorem 1]) shows that the answer to such questions (and several other variants of them) is "no" (see Corollary 22). When combined with Theorem 14, Proposition 23, Proposition 24, and Proposition 26. this will allow us to obtain the "complete solution" promised in the introduction (see Theorem 27). 
Theorem 21 (Solecki). Let $\mathcal{I}$ be a family of closed subsets of a Polish space T. Let $A$ be an analytic subset of $T$. Then one of the following conditions holds.

(1) $A \subseteq \bigcup \mathcal{J}$ for some countable $\mathcal{J} \subseteq \mathcal{I}$.

(2) There exists a $\mathrm{G}_{\delta}$ subset $G$ of $T$ such that $G \subseteq A$ and $G \nsubseteq \bigcup \mathcal{J}$ for every countable $\mathcal{J} \subseteq \mathcal{I}$.

Corollary 22. Let $X$ be a space with the $\operatorname{PSP}\left(\mathrm{G}_{\delta}\right)$. Then $X$ has the $\operatorname{PSP}$ (analytic).

Proof. Without loss of generality, assume that $X$ is a subspace of $T=[0,1]^{\omega}$. Define

$$
\mathcal{I}=\left\{K \subseteq T: K \text { is closed in } T \text { and }|X \cap K|<\omega_{1}\right\} .
$$

Let $A$ be an analytic subset of $T$ such that $A \cap X$ is uncountable. Since $\omega_{1}$ has uncountable cofinality, condition (1) in Theorem 21 cannot hold. Therefore, condition (2) must hold for some $\mathrm{G}_{\delta}$ subset $G$ of $T$. In particular $G \cap X$ is uncountable, so it contains a copy of $2^{\omega}$ by the $\operatorname{PSP}\left(\mathrm{G}_{\delta}\right)$. Since $G \subseteq A$, it follows that $A \cap X$ contains a copy of $2^{\omega}$, which is what we needed to show.

The following proposition answers a question that appeared in an earlier version of this article.

Proposition 23 (Miller). There exists a space $X$ that has the PSP(analytic) but not the PSP(coanalytic).

Proof. Let $W O \subseteq \omega^{\omega}$ be the set of codes of well-orderings of $\omega$, as in [2, page 485]. For each infinite $\alpha<\omega_{1}$, let $z_{\alpha} \in$ WO be a code for a well-ordering of $\omega$ of type $\alpha$. Define $Z=\left\{z_{\alpha}: \omega \leq \alpha<\omega_{1}\right\}$ and $X=Z \cup\left(\omega^{\omega} \backslash\right.$ WO $) \subseteq \omega^{\omega}$. By [2, Lemma 25.9], WO is a coanalytic subset of $\omega^{\omega}$, so $X \cap W O=Z$ is an uncountable coanalytic subset of $X$. Since the Boundedness Lemma (see 2, Corollary 25.14]) implies that the only analytic subsets of $\omega^{\omega}$ contained in $Z$ are the countable ones, $Z$ does not contain any copy of $2^{\omega}$. Therefore, $X$ does not have the PSP(coanalytic).

In order to prove that $X$ has the PSP(analytic), let $A$ be an analytic subset of $\omega^{\omega}$ such that $A \cap X$ is uncountable. Notice that $A \cap\left(\omega^{\omega} \backslash\right.$ WO $)$ must be uncountable, otherwise $A \cap \mathrm{WO}=A \backslash\left(A \cap\left(\omega^{\omega} \backslash \mathrm{WO}\right)\right)$ would be an uncountable analytic subset of $\omega^{\omega}$ that has uncountable intersection with $Z$, contradicting the Boundedness Lemma. Now it remains to apply Theorem 2 .

Proposition 24. There exists a space $X$ that has the PSP(open) but not the PSP(closed).

Proof. Let $B$ be a Bernstein set in $[0,1]$. Define

$$
X=(B \times\{1\}) \cup([0,1] \times[0,1)) \subseteq[0,1] \times[0,1] .
$$

Notice that $B$ is an uncountable closed subset of $X$ containing no copies of $2^{\omega}$. Therefore $X$ does not have the PSP(closed). To see that $X$ has the PSP(open), observe that every non-empty open subset of $X$ contains a copy of $[0,1] \times[0,1]$, hence a copy of $2^{\omega}$.

The following proposition, which is essentially due to Medini and Milovich (see [6. Theorem 28]), shows that the space given by Proposition 24 can even be made zerodimensional. Next, we recall some terminology that will be used in its proof. Every filter is assumed to be on $\omega$. Furthermore, we will assume that Cof $\subseteq \mathcal{F} \subsetneq \mathcal{P}(\omega)$ for every filter $\mathcal{F}$, where $\operatorname{Cof}=\{x \subseteq \omega:|\omega \backslash x|<\omega\}$. Recall that $\mathcal{C} \subseteq \mathcal{P}(\omega)$ has the finite intersection property if $\cap F$ is infinite whenever $F$ is a non-empty finite 
subset of $\mathcal{C}$. Given $x \subseteq \omega$, define $x^{0}=x$ and $x^{1}=\omega \backslash x$. Recall that $\mathcal{A} \subseteq \mathcal{P}(\omega)$ is an independent family if $\bigcap\left\{x^{\nu(x)}: x \in F\right\}$ is infinite whenever $F$ is a non-empty finite subset of $\mathcal{A}$ and $\nu: F \longrightarrow 2$.

Proposition 25. There exists a subspace $X$ of $2^{\omega}$ that has the PSP(open) but not the PSP(closed).

Proof. Throughout this proof, we will identify $\mathcal{P}(\omega)$ with $2^{\omega}$ via characteristic functions. In particular, we will identify every filter and every independent family with a subspace of $2^{\omega}$.

First, we will show that every filter $\mathcal{F} \subseteq 2^{\omega}$ has the PSP(open). This is trivial if $\mathcal{F}=$ Cof, so assume that $\mathcal{F} \supsetneq$ Cof. Let $U$ be an uncountable open subset of $\mathcal{F}$. In particular $U \neq \varnothing$, so $[s] \cap \mathcal{F} \subseteq U$ for some $s \in{ }^{<\omega_{2}}$. Now pick any coinfinite $z \in \mathcal{F}$ such that $z\left\lceil\operatorname{dom}(s)=s\right.$. It is easy to see that $\{x \in[s]: z \subseteq x\}$ is a copy of $2^{\omega}$ contained in $U$.

By [6. Lemma 7], there exists an independent family $\mathcal{A} \subseteq 2^{\omega}$ that is homeomorphic to $2^{\omega}$. In particular, we can fix a Bernstein set $B$ in $\mathcal{A}$. Since $\mathcal{A}$ is an independent family, the collection

$$
\mathcal{C}=B \cup\{\omega \backslash x: x \in \mathcal{A} \backslash B\}
$$

has the finite intersection property (actually, it is an independent family). Therefore, there exists a filter $\mathcal{F}$ such that $\mathcal{C} \subseteq \mathcal{F}$. Let $X=\mathcal{F}$. It is easy to realize that $B$ is an uncountable closed subset of $X$ that does not contain any copy of $2^{\omega}$. In particular, $X$ does not have the PSP(closed).

Proposition 26. There exists a space $X$ that has the PSP(clopen) but not the PSP(open).

Proof. Let $B$ be a Bernstein set in $[0,1] \times[0,1)$. Define

$$
X=B \cup([0,1] \times\{1\}) \subseteq[0,1] \times[0,1] .
$$

Notice that $B$ is an uncountable open subset of $X$ containing no copies of $2^{\omega}$. Therefore $X$ does not have the PSP(open). On the other hand, the fact that $B$ is connected (use the same argument as in the proof of [7, Lemma 3.2]) implies that $X$ is connected. Since $X$ contains a copy of $[0,1]$, hence a copy of $2^{\omega}$, it follows that $X$ has the PSP(clopen).

Notice that the space $X$ given by Proposition 26 cannot be zero-dimensional because every open set in a zero-dimensional space can be written as a countable union of clopen sets.

Theorem 27. Consider the following conditions on a space $X$.

(1) $X$ has the PSP(coanalytic).

(2) $X$ has the PSP(analytic).

(3) $X$ has the $\operatorname{PSP}\left(\mathrm{G}_{\delta}\right)$.

(4) $X$ has the $\operatorname{PSP}\left(\mathrm{F}_{\sigma}\right)$.

(5) $X$ has the PSP(closed).

(6) $X$ has the PSP(open).

(7) $X$ has the PSP(clopen).

The implications (11) $\rightarrow$ (2) $\leftrightarrow$ (3) $\rightarrow$ (4) $\leftrightarrow$ (5) $\rightarrow$ (6) $\rightarrow$ (7) hold for every $X$. The implication (3) $\leftarrow$ (4) holds for every $X$ if and only if $\mathfrak{b}>\omega_{1}$. There exist ZFC counterexamples to the implications (1) $\leftarrow$ (2), (5) $\leftarrow$ (6), and (6) $\leftarrow$ (7). 
Proof. The implications (3) $\rightarrow$ (5) and (4) $\rightarrow$ (6) $\rightarrow$ (7) are trivial. The equivalence (2) $\leftrightarrow$ (3) follows from Corollary 22, and it shows that the implication (11) $\rightarrow$ (2) holds. The equivalence (4) $\leftrightarrow$ (5) holds because $\omega_{1}$ has uncountable cofinality. This concludes the proof of the first statement. Now it is easy to realize that the second statement follows from Theorem 14. Finally, the third statement is the content of Proposition 23, Proposition 24, and Proposition 26.

\section{Generalizing the Grinzing Property}

In this section, we will define a natural generalization of the Grinzing property and extend some of the results from Section 2. While Question 1 below seems to be of independent interest, it will turn out to be relevant in the next section as well (see the remark preceding Question 3).

Definition 28. Fix cardinals $\kappa, \lambda$ such that $\omega_{1} \leq \kappa \leq \mathfrak{c}$ and $\lambda \leq \kappa$. We will say that a subset $W$ of $2^{\omega}$ has the $(\kappa, \lambda)$-Grinzing property (briefly, the $(\kappa, \lambda)$-GP) if $|W| \geq \kappa$ and for every $Y \subseteq W$ such that $|Y| \geq \kappa$ there exist subsets $Y_{\alpha}$ of $Y$ for $\alpha \in \lambda$ such that $\left|Y_{\alpha}\right| \geq \kappa$ for each $\alpha$ and $\operatorname{cl}\left(Y_{\alpha}\right) \cap \mathrm{cl}\left(Y_{\beta}\right)=\varnothing$ whenever $\alpha \neq \beta$, where the closure is taken in $2^{\omega}$.

Notice that a subset $W$ of $2^{\omega}$ such that $|W| \geq \kappa$ has the $(\kappa, \lambda)$-GP if and only if every subset of $W$ of size $\kappa$ has the $(\kappa, \lambda)$-GP. Also notice that the $(\kappa, \lambda)$-GP gets stronger as $\lambda$ gets bigger. Furthermore, it is clear that the $\left(\omega_{1}, \omega_{1}\right)$-GP is simply the GP.

Using the well-known Lemma 30, it is easy to show that $2^{\omega}$ has the $(\kappa, \omega)$-GP for every cardinal $\kappa \leq \mathfrak{c}$ of uncountable cofinality. The following proposition shows that the restriction on the cofinality is really necessary.

Proposition 29. Let $\kappa$ be a cardinal of countable cofinality such that $\omega_{1}<\kappa<\mathfrak{c}$. Then no subset of $2^{\omega}$ has the $(\kappa, 2)-\mathrm{GP}$.

Proof. Fix cardinals of uncountable cofinality $\kappa_{n}<\kappa$ for $n \in \omega$ such that $\kappa_{n} \rightarrow \kappa$. Let $W$ be a subset of $2^{\omega}$ such that $|W|=\kappa$. Fix a compatible metric on $2^{\omega}$. Using Lemma 30, it is possible to obtain subsets $Y_{n}$ of $W$ for $n \in \omega$ such that $\left|Y_{n}\right|=\kappa_{n}$ and $\operatorname{diam}\left(Y_{n}\right) \rightarrow 0$ as $n \rightarrow \infty$. Pick $z_{n} \in Y_{n}$ for each $n$. By compactness, there exists $z \in 2^{\omega}$ and a subsequence of $\left\langle z_{n}: n \in \omega\right\rangle$ that converges to $z$. It is easy to check that the corresponding subsequence of $\left\langle Y_{n}: n \in \omega\right\rangle$ converges to $z$. Assume without loss of generality that $\left\langle Y_{n}: n \in \omega\right\rangle$ converges to $z$. This implies $z \in \operatorname{cl}(Z)$ for every $Z \subseteq Y=\bigcup_{n \in \omega} Y_{n}$ of size $\kappa$, where the closure is taken in $2^{\omega}$. In particular, $Y$ witnesses that $W$ does not have the $(\kappa, 2)$-GP.

Lemma 30. Let $\kappa$ be a cardinal of uncountable cofinality. Then every space of size at least $\kappa$ has a $\kappa$-crowded subspace.

Proof. Let $X$ be a space of size at least $\kappa$. Define

$$
\mathcal{U}=\{U \subseteq X: U \text { is open in } X \text { and }|U|<\kappa\} .
$$

and $V=\bigcup \mathcal{U}$. It is obvious that $\mathcal{U}$ is a cover of $V$. Let $\mathcal{V}$ be a countable subcover of $\mathcal{U}$. Notice that $|V|<\kappa$ because $V=\bigcup \mathcal{V}$ and $\kappa$ has uncountable cofinality. So $Y=X \backslash V$ is non-empty. Furthermore, it is clear that every non-empty open subset of $Y$ has size at least $\kappa$.

The following fundamental question is open. Notice that Corollary 11 gives a positive answer in the case $(\kappa, \lambda)=\left(\omega_{1}, \omega_{1}\right)$. 
Question 1. For which cardinals $\kappa, \lambda$ such that $\omega_{1} \leq \lambda \leq \kappa \leq \mathfrak{c}$ and $\kappa$ has uncountable cofinality is it possible to prove in ZFC that there exists a subset of $2^{\omega}$ with the $(\kappa, \lambda)$-GP?

The following result (see [10, Section 4]), together with Proposition 12] shows that the statement " $2 \omega$ has the $(\mathfrak{c}, \mathfrak{c})$-GP" is independent of ZFC.

Theorem 31 (Miller). It is consistent that for every $Y \subseteq 2^{\omega}$ of size $\mathfrak{c}$ there exists a continuous map $f: 2^{\omega} \longrightarrow 2^{\omega}$ such that $f[Y]=2^{\omega}$.

Corollary 32. It is consistent that $2^{\omega}$ has the $(\mathfrak{c}, \mathfrak{c})$-GP.

Proof. Use the fact that $2^{\omega} \approx 2^{\omega} \times 2^{\omega}$.

On the other hand, the proofs of Proposition 12 and Theorem 13 can easily be adapted to obtain the following results.

Proposition 33. Assume $\mathfrak{b}=\kappa$. Then $2^{\omega}$ does not have the $\left(\kappa, \omega_{1}\right)$-GP.

Theorem 34. Assume MA. Then $2^{\omega}$ has the $(\kappa, \kappa)$-GP for every cardinal $\kappa<\mathfrak{c}$ of uncountable cofinality.

\section{Generalizing the perfect set property}

We begin by stating the following natural generalization of the $\operatorname{PSP}(\boldsymbol{\Gamma})$.

Definition 35. Fix an uncountable cardinal $\kappa$. Let $X$ be a space and $\boldsymbol{\Gamma}$ a pointclass. We will say that $X$ has the $\kappa$-perfect set property for $\boldsymbol{\Gamma}$ subsets (briefly, the $\kappa$-PSP $(\boldsymbol{\Gamma}))$ if for every $\boldsymbol{\Gamma}$ subset $A$ of $X$ either $|A|<\kappa$ or $A$ contains a copy of $2^{\omega}$.

Notice that the $\kappa-\operatorname{PSP}(\boldsymbol{\Gamma})$ gets stronger as $\kappa$ gets smaller and as $\boldsymbol{\Gamma}$ gets bigger. Furthermore, it is clear that the $\omega_{1}-\operatorname{PSP}(\boldsymbol{\Gamma})$ is simply the $\operatorname{PSP}(\boldsymbol{\Gamma})$.

The following is the most general question that we can imagine on this subject.

Question 2. What is the status of the statement "For every space $X$, if $X$ has the $\kappa$-PSP $(\boldsymbol{\Gamma})$ then $X$ has the $\kappa^{\prime}$-PSP $\left(\boldsymbol{\Gamma}^{\prime}\right)$ " as $\kappa, \kappa^{\prime}$ range over all uncountable cardinals and $\boldsymbol{\Gamma}, \boldsymbol{\Gamma}^{\prime}$ range over all pointclasses?

Theorem 27 can be viewed as a partial answer to the above question, in the case where $\kappa=\kappa^{\prime}=\omega_{1}$ and $\boldsymbol{\Gamma}, \boldsymbol{\Gamma}^{\prime}$ are at most analytic. In the rest of this section, we will essentially point out further concrete instances of Question 2 which seem particularly interesting.

The following two results generalize Proposition 15] and Corollary 22 from $\omega_{1}$ to an arbitrary uncountable cardinal $\kappa$. Lemma 38 will allow us to handle the case in which $\kappa$ has countable cofinality.

Proposition 36. Assume $\mathfrak{b}>\kappa$, where $\kappa$ is an uncountable cardinal. Then the $\kappa$-PSP(closed) implies the $\kappa$-PSP(analytic) for every space.

Proof. If $\kappa$ has uncountable cofinality, the desired result follows from a straightforward adaptation of the proof of Proposition 15. So assume that $\kappa$ has countable cofinality, and let $X$ be a space with the $\kappa$-PSP(closed). By Lemma 38, there exists a cardinal $\kappa^{\prime}<\kappa$ of uncountable cofinality such that $X$ has the $\kappa^{\prime}$-PSP(closed). Since $\mathfrak{b}>\kappa^{\prime}$, it follows that $X$ has the $\kappa^{\prime}$-PSP(analytic), which obviously implies the $\kappa$-PSP(analytic). 
Proposition 37. Let $\kappa$ be an uncountable cardinal. Then the $\kappa-\mathrm{PSP}\left(\mathrm{G}_{\delta}\right)$ implies the $\kappa$-PSP(analytic) for every space.

Proof. If $\kappa$ has uncountable cofinality, the desired result follows from a straightforward adaptation of the proof of Corollary 22. Otherwise, apply Lemma 38 as in the proof of Proposition 36 .

Lemma 38. Assume that $\boldsymbol{\Gamma}$ is a pointclass such that $2^{\omega}$ has the $\mathfrak{c}-\operatorname{PSP}(\boldsymbol{\Gamma})$. Let $\kappa$ be an uncountable cardinal of countable cofinality. Assume that $X$ is a space with the $\kappa-\operatorname{PSP}(\boldsymbol{\Gamma})$. Then there exists a cardinal $\kappa^{\prime}<\kappa$ of uncountable cofinality such that $X$ has the $\kappa^{\prime}-\mathrm{PSP}(\boldsymbol{\Gamma})$.

Proof. Assume without loss of generality that $X$ is a subspace of $T=[0,1]^{\omega}$. Fix a compatible metric on $T$. Fix cardinals of uncountable cofinality $\kappa_{n}<\kappa$ for $n \in \omega$ such that $\kappa_{n} \rightarrow \kappa$. Assume, in order to get a contradiction, that there exist $\boldsymbol{\Gamma}$ subsets $A_{n}$ of $X$ for $n \in \omega$ such that $\left|A_{n}\right| \geq \kappa_{n}$ for each $n$ and no $A_{n}$ contains a copy of $2^{\omega}$. Define $A=\bigcup_{n \in \omega} A_{n}$. First assume that the pointclass $\boldsymbol{\Gamma}$ is closed under countable unions. Then $A$ is a $\boldsymbol{\Gamma}$ subset of $X$ of size at least $\kappa$, so it contains a copy $K$ of $2^{\omega}$ by the $\kappa-\operatorname{PSP}(\boldsymbol{\Gamma})$. Notice that $\left|K \cap A_{n}\right|=\mathfrak{c}$ for some $n$ because $|K|=\mathfrak{c}$ has uncountable cofinality. The fact that $K \approx 2^{\omega}$ has the c-PSP $(\boldsymbol{\Gamma})$ concludes the proof in this case.

Now assume $\boldsymbol{\Gamma}=\boldsymbol{\Pi}_{\xi}^{0}$, where $\xi$ is a successor ordinal such that $1 \leq \xi<\omega_{1}$. Choose $A_{n}$ as above, but require in addition that $\operatorname{diam}\left(A_{n}\right) \rightarrow 0$ as $n \rightarrow \infty$. This is possible by Lemma 30, Pick $z_{n} \in A_{n}$ for each $n$. By compactness, there exists $z \in T$ and a subsequence of $\left\langle z_{n}: n \in \omega\right\rangle$ that converges to $z$. It is easy to check that the corresponding subsequence of $\left\langle A_{n}: n \in \omega\right\rangle$ converges to $z$. Assume without loss of generality that $\left\langle A_{n}: n \in \omega\right\rangle$ converges to $z$. Define $A=\bigcup_{n \in \omega} A_{n}$. In the case $\xi>1$, it is not hard to show that $A$ is a $\Gamma$ subset of $X$ of size at least $\kappa$, which yields a contradiction as before. In the case $\xi=1$, consider $A \cup(\{z\} \cap X)$ instead.

The following question is obviously inspired by Proposition 19] By inspecting its proof, it is not hard to realize that the answer would be "yes" assuming the existence of a subset of $2^{\omega}$ with the $\left(\kappa, \omega_{1}\right)$-GP (see Question 1).

Question 3. Does $\mathfrak{b}=\kappa$ imply that there exists a space with the $\kappa$-PSP(closed) but not the $\kappa$-PSP(analytic)?

Notice that an affirmative answer to Question 3, combined with Proposition 36 . Proposition 37 and Lemma 38, would allow us to generalize Theorem 27 from $\omega_{1}$ to an arbitrary uncountable cardinal $\kappa$.

Finally, we observe that Corollary 40 below shows that another concrete instance of Question 2] is settled by the following classical result (see [4, Proposition 13.7]).

Theorem 39 (Sierpiński). Every $\boldsymbol{\Sigma}_{2}^{1}$ subset of a Polish space $T$ can be written as $\bigcup_{\alpha \in \omega_{1}} A_{\alpha}$, where each $A_{\alpha}$ is a Borel subset of $T$.

Corollary 40. Assume that $X$ is a space with the $\omega_{2}-\mathrm{PSP}\left(\mathrm{G}_{\delta}\right)$. Then $X$ has the $\omega_{2}-\operatorname{PSP}\left(\boldsymbol{\Sigma}_{2}^{1}\right)$

Proof. Let $A$ be a $\Sigma_{2}^{1}$ subset of $X$ such that $|A| \geq \omega_{2}$. By Theorem 39] it is possible to write $A=\bigcup_{\alpha \in \omega_{1}} A_{\alpha}$, where each $A_{\alpha}$ is a Borel subset of $X$. Fix $\alpha$ such that $\left|A_{\alpha}\right| \geq \omega_{2}$. Notice that $X$ has the $\omega_{2}$-PSP(analytic) by Proposition 37 In particular, $A_{\alpha}$ contains a copy of $2^{\omega}$. 


\section{ACKNowledgements}

The author is very grateful to Arnie Miller for realizing that an early "proof" of Corollary 11 used an assumption that is not provable in ZFC, and for kindly "donating" to him its current proof and Proposition 23. He also thanks Ken Kunen for contributing to the proof of Proposition 19, as explained in the remark that follows it. Finally, he thanks Lyubomyr Zdomskyy for several discussions on the topic of this article, and in particular for suggesting Lemma 18 and the remarks on Luzin sets (namely, the second proof of Proposition 12 and Proposition 20).

\section{REFERENCES}

[1] F. Galvin, A. W. Miller. $\gamma$-sets and other singular sets of real numbers. Topology Appl. 17:2 (1984), 145-155.

[2] T. JECH. Set theory. The third millennium edition, revised and expanded. Springer Monographs in Mathematics. Springer-Verlag, Berlin, 2003. xiv+769 pp.

[3] H. Judah, S. Shelah. Killing Luzin and Sierpiński sets. Proc. Amer. Math. Soc. 120:3 (1994), 917-920.

[4] A. Kanamori. The higher infinite. Second edition. Paperback reprint of the 2003 edition. Springer Monographs in Mathematics. Springer-Verlag, Berlin, 2009. xxii+536 pp.

[5] A. S. Kechris. Classical descriptive set theory. Graduate Texts in Mathematics, 156. Springer-Verlag, New York, 1995. xviii+402 pp.

[6] A. Medini, D. Milovich. The topology of ultrafilters as subspaces of $2^{\omega}$. Topology Appl. 159:5 (2012), 1318-1333.

[7] J. van Mill. Strong local homogeneity does not imply countable dense homogeneity. Proc. Amer. Math. Soc. 84:1 (1982), 143-148.

[8] J. VAN MiLl. The infinite-dimensional topology of function spaces. North-Holland Mathematical Library, 64. North-Holland Publishing Co., Amsterdam, 2001. xii+630 pp.

[9] A. W. Miller. On the length of Borel hierarchies. Ann. Math. Logic. 16:3 (1979), 233-267.

[10] A. W. Miller. Mapping a set of reals onto the reals. J. Symbolic Logic. 48:3 (1983), 575-584.

[11] A. W. Miller. Projective subsets of separable metric spaces. Ann. Pure Appl. Logic. 50:1 (1990), 53-69.

[12] S. SolECKI. Covering analytic sets by families of closed sets. J. Symbolic Logic. 59:3 (1994), $1022-1031$.

Kurt Gödel Research Center for Mathematical Logic

University of Vienna, Währinger Strasse 25, A-1090 Wien, Austria

E-mail address: andrea.medini@univie.ac.at

URL: http://www.logic.univie.ac.at/〜medinia2/ 